\title{
Development of a Prognosis Nomogram of Treatment Outcomes for MDR-tuberculosis in Guinea (Conakry): A Retrospective Cohort Analysis
}

\author{
Boubacar Djelo Diallo ${ }^{1}$, , Alhassane Diallo ${ }^{1}$, Lansana Mady Camara ${ }^{1}$, Mafouné Diallo ${ }^{1}$, \\ Souleymane Camara ${ }^{1}$, Boubacar Bah ${ }^{3}$, Magassouba Aboubacar Sidiki , Alpha Oumar Barry ${ }^{1}$, \\ Thierno Hassane Diallo ${ }^{1}$, Oumou Younoussa Sow ${ }^{1}$ \\ ${ }^{1}$ Department of Pneumo-phtisiology, Ignace Deen National Hospital, Faculty of Health Sciences and Techniques, Gamal Abdel Nasser \\ University of Conakry, CHU Conakry, Guinea \\ ${ }^{2}$ National Tuberculosis Control Program, Conakry, Guinea \\ ${ }^{3}$ Carrière Tuberculosis Center, Conakry, Guinea
}

Email address:

diallodjelo@yahoo.fr (B. D. Diallo)

${ }^{*}$ Corresponding author

\section{To cite this article:}

Boubacar Djelo Diallo, Alhassane Diallo, Lansana Mady Camara, Mafouné Diallo, Souleymane Camara, Boubacar Bah, Magassouba Aboubacar Sidiki, Alpha Oumar Barry, Thierno Hassane Diallo, Oumou Younoussa Sow. Development of a Prognosis Nomogram of Treatment Outcomes for MDR-tuberculosis in Guinea (Conakry): A Retrospective Cohort Analysis. Central African Journal of Public Health. Vol. 6, No. 1, 2020, pp. 33-41. doi: 10.11648/j.cajph.20200601.16

Received: December 18, 2019; Accepted: January 2, 2020; Published: January 16, 2020

\begin{abstract}
Despite the availability of the drug treatment for tuberculosis (TB) more than 75 years, mortality and drug resistance are increasing. Therefore, little data is available in Guinea. We aimed to develop and validate a prognosis nomogram of MDR-TB treatment outcomes. A retrospective cohort study was conducted among men and women, aged 18 years or older, with MDR-TB, from three major drug-resistance TB centres in Guinea. We used the logistic regression to analyse treatment outcomes. Prognostic factors with a $p$ value less than 0.05 from a multivariate model were used to build nomogram and assessed their performance based on discriminative c-index, and calibration using the Hosmer-Lemeshow (H-L) test. To derive the optimal cut-off point score, the Youden's index method was used. Among 232 patients with MDR-TB enrolled and followed between June 07, 2016 and June 22, 2018, 218 were analyzed. All patients were resistant to rifampicin, which diagnosed by the Xpert MTB/RIF. The overall rate of success was $73 \%$. Factors associated with successful treatment in drugresistant TB patients were higher BMI more than $18.5 \mathrm{~kg} / \mathrm{m}^{2}(\mathrm{p}=0.0253$; aOR $=2.94)$, good adherence to treatment $(\mathrm{p}=<$ 0.0001; aOR $=33.92)$, normal platelets count $(\mathrm{p}=0.0053$; $\mathrm{OR}=1.004)$, and the absence of clinical symptoms such as chest pain $(\mathrm{p}=0.0083$; aOR $=3.19)$ and depression $(\mathrm{p}=0.0308 ; \mathrm{aOR}=8.62)$. The discrimination ( $c$-index $=0.848[95 \%$ bootstrap $\mathrm{CI}, 0.780-0.916]$ in the derivation sample and 0.803 after correction for optimism) and calibration $\left(\mathrm{H}-\mathrm{L}_{\mathrm{X}}{ }^{2}=2.91[\mathrm{p}=0.94]\right)$ were good. The optimal absolute risk threshold was $20 \%$, corresponding to a sensibility of $95 \%$ and specificity of $58 \%$. Treatment success outcomes was lower than those recommended by the World Health Organization (75\%). We recommend to improve the MDR-TB patient monitoring during treatment, nutritional status, and considering the psychological state. Our prognosis nomogram needs to be validated in an external population before it can be used in clinical practice.
\end{abstract}

Keywords: Nomogram, Multidrug-resistant, Tuberculosis, Guinea

\section{Background}

Despite the availability of the drug treatment for tuberculosis (TB) since 1943, mortality and drug resistance are increasing, mainly because the emergence of the HIV infection, the poor prevention programs, and the persisting global poverty [1].

Multidrug-resistant (MDR)-TB is defined as resistance at least to isoniazid (INH) and rifampicin (R) with an estimated 
600000 new cases reported by the World Health Organization (WHO) in 2016 [2]. While Russia, China, and India accounted $47 \%$ of the global incident cases of MDRTB [2], little data is available in Guinea. Nevertheless, according to the national tuberculosis program reports, the number of new incident cases of MDR-TB has increased from 53 in 2008 to 265 cases in 2018 [3].

Treatment outcomes of MDR tuberculosis patients and factors associated with these outcomes have been done in several studies [4-11]. While an estimated success (cured and completed treatment) rate of treatment regimen ranged from $53.4 \%$ in Morocco [5] to 87.3 in Rwanda [10], results about predictors of treatment outcomes are contradictory. A recent meta-analysis identified negative HIV infection and non-use alcohol misuse as predictors associated with successful treatment [12].

An easy-to-use prognosis nomogram is need to help the identification of the patients with high chance of successful treatment, especially in low income countries. To our knowledge, no study has been conducted to develop prognosis nomogram of MDR-TB treatment outcomes. Here, we reported data from a retrospective cohort study of patient treated with a standardized shorted treatment regimen of 9months in Guinea. We aimed to develop and validate a prognosis nomogram of MDR-TB treatment outcomes during follow-up

\section{Methods}

\subsection{Study Design and Population}

We retrospectively analyzed 218 patients with MDR-TB enrolled between June 07, 2016 and June 22, 2018 in the multicenter longitudinal cohort study conducted in three major drug-resistance TB centres in Guinea (Ignace-Deen, Carrière and Tombolia). For MDR-TB diagnosis, both sputum smear and culture were performed in these centres. Patients were seen at baseline and followed by monthly visit for 9 months according to the WHO standardized $9-12$ months shorter treatment regimen guidelines recommendations [13]. Patients who were younger than 18 years were excluded from the analysis. Ethical approval was obtained from the Guinean Ethics Committee for Heath Research.

\subsection{Bacteriology, and Drug Susceptibility Testing}

The diagnosis of drug-resistance was done by the Xpert MTB/RIF test. For others anti-TB drugs, the drug susceptibility testing (DST), based on solid culture (Lowenstein-Jensen) were done late, and in a very partial way. Second-line anti-TB drugs were not routinely tested. Xpert MTB/RIF test and DST were only available at the main TB centre located in Ignace Deen.

\subsection{Treatment Regimens for MDR-TB in Guinea}

According to the guideline for the MDR management in Guinea [3], naive patients for second-line anti-TB drugs were treated with a short 9-month regimen, consisting of an intensive phase lasting a minimum of 4 months including moxifloxacin, kanamycin, clofazimine, prothionamide, pyrazinamide, ethambutol, and INH at high dose. The intensive phase was then followed by the continuation phase during 5 months and consisting of administration of four drugs: moxifloxacin, clofazimine, pyrazinamide, and ethambutol. Sputum smears and cultures were obtained monthly during the MDR-TB treatment duration.

\subsection{Data Collection}

Data was collected using a case report form (CRF) from the MDR-TB registry that containing sociodemographic, clinical data, and the laboratory test results (sputum smear or culture conversion) for all patients who were admitted in the three major TB centres outpatient care. Additional information was completed with data from the patients' clinical files. The following clinical and demographic data record were extracted: age, gender, residence, comorbidity, HIV status, history of the previously treated TB, the presence of cavities on chest X-ray determined by the senior radiologist, baseline data on weight, sputum smear and culture, clinical symptoms (as chest pain or cough), and biological data. Additionally, we extracted depression status where the patient was asked if he/she was depressed or anxious, and adherence to MDR-TB treatment status during follow-up based on the proportion of days covered (PDC). For each patient, we calculated a PDC by dividing the number of days covered with MDR-TB treatment delivered over one month by 31 . Then, we considered a minimal value of PDC during follow-up for each patient as a marker of adherence to MDR-TB treatment. Conventionally, the PDC was dichotomized between good adherence if the PDC was 0.8 or more and poor adherence otherwise [14].

\subsection{Treatment Outcomes}

Using the revised WHO recommendations in 2013 [15], we classified the treatment outcomes into two categories (successful or unsuccessful). Successful treatment outcomes corresponded to patient who declared as either "cured" or "treatment completed". Unsuccessful treatment outcomes included "treatment failure", or "death form any reason" or "lost to follow-up" or "not evaluated".

\subsection{Statistical Analysis}

Frequencies (percentage) or means (standard deviation; SD) were used to describe categorical and continuous variables at baseline. Univariate logistic regression was used to identify prognostic factors associated with successful MDR-TB treatment, and then candidates with a p-value less than 0.10 were entered into the multivariate logistic regression. The independent predictors of successful MDR-TB treatment from multivariate regression were selected through a backward procedure based on the lowest Akaike information criterion. Odds ratio (OR) together with their $95 \%$ confidence interval (CI) were used as association measures. 
We constructed a nomogram that included the selected prognostic factors from final logistic model to estimate the probability of successful MDR-TB treatment at 9-months. A raw prognostic score was computed by summing the contribution of each individual factor, based on the estimate for each factor in the nomogram. Then, we divided patients into four prognostic chance groups: "low chance", "moderate chance", "intermediate chance", and "high chance". We did calibration plots with the Hosmer and Lemeshow statistic test [16], and computed the discriminative $c$-index to assess the performance of the nomogram. 1,000 random samples of the population were used to drive the $95 \%$ CI bootstrap percentile for the c-index, and to correct the optimism. We used the receiver-operating characteristic (ROC), applying the Youden's index method [17], to derive the optimal cut-off point score. Then, at this optimal threshold, the performance measures including the sensitivity, specificity, and positive and negative predictive values (PV) were estimated. The final model was internally validated using the 1,000 samples bootstrap procedure. All data analyses were done in $\mathrm{R}$ (version 3.5.1). Significance was defined as a p-value less than 0.05 , and all tests were two-sided.

\section{Results}

Socio-demographic and clinical characteristics at baseline

A total of 218 patients with MDR-TB who meet the inclusion criterion were analyzed. Among them, 18 (8.2\%) patients were enrolled in the second half of 2016, 136 $(62.4 \%)$ patients enrolled in 2017 , and $64(29.4 \%)$ in the first half of 2018. The mean age of these patients was $33.7 \pm 11.3$ years, with the majority of them were male $(68 \%)$ and leaving in urban area (78\%). Most patients presented cough $(94 \%)$ or night sweat $(66 \%), 50(23 \%)$ patients were HIV positive, $83(41 \%)$ patients had a BIM above $18.5 \mathrm{~kg} / \mathrm{m}^{2}$, only $10(5 \%)$ patients reported depression. Au total 181 $(83 \%)$ patients had available X-ray film for interpretation and 17 (9\%) of among them had showed cavitary lesions. According to the history of TB, 179 (82\%) patients were previously treated, $192(88 \%)$ and $176(81 \%)$ had a positive sputum smear and culture at baseline respectively, and all patients had resistance to rifampicin (Table 1).

Table 1. Population characteristics at baseline.

\begin{tabular}{|c|c|c|c|}
\hline \multirow{3}{*}{ Characteristics } & \multirow{2}{*}{ Total } & \multicolumn{2}{|c|}{ Treatment outcomes (n,\%) } \\
\hline & & Successful & Unsuccessful \\
\hline & $(n=218)$ & $(n=159)$ & $(n=59)$ \\
\hline Gender (Male) & $149(68.35)$ & $114(76.51)$ & $35(23.49)$ \\
\hline Age (years, $\mathrm{m} \pm \mathrm{SD}$ ) & $33.7(11.3)$ & $32.8(10.4)$ & $36.3(13.2)$ \\
\hline Residence (Urban) & $169(77.52)$ & $118(69.82)$ & $51(30.18)$ \\
\hline $\operatorname{BMI}\left(>18.5 \mathrm{~kg} / \mathrm{m}^{2}\right)$ & $89(40.83)$ & $64(83.12)$ & $13(16.88)$ \\
\hline \multicolumn{4}{|l|}{ Years of inclusion } \\
\hline 2017 & $136(62.39)$ & $102(75.00)$ & $34(25.00)$ \\
\hline 2018 & $64(29.36)$ & $45(70.31)$ & $19(29.69)$ \\
\hline HIV status (Positive) & $50(23.15)$ & $31(62.00)$ & $19(38.00)$ \\
\hline History of TB treatment (Previously treated) & $179(82.49)$ & $135(7.42)$ & $44(24.58)$ \\
\hline Sputum smear (Positive) & $192(88.07)$ & $144(75.00)$ & $48(25.00)$ \\
\hline Sputum culture (Positive) & $176(80.73)$ & $132(75.00)$ & $44(25.00)$ \\
\hline Lung Cavities on X-ray (Yes) & $17(9.39)$ & $12(70.59)$ & $5(29.41)$ \\
\hline Chest pain (Yes) & $86(39.45)$ & $29(21.97)$ & $30(34.88)$ \\
\hline Night sweat (Yes) & $120(55.05)$ & $92(76.67)$ & $28(23.33)$ \\
\hline Nausea (Yes) & $15(6.88)$ & $8(53.33)$ & $7(46.67)$ \\
\hline Vomiting (Yes) & $22(10.09)$ & $11(50.00)$ & $11(50.00)$ \\
\hline Leukocytes count $(\mathrm{m} \pm \mathrm{SD})$ & $7.9(3.5)$ & $8.0(3.8)$ & $7.4(2.7)$ \\
\hline Hemoglobin count $(\mathrm{m} \pm \mathrm{SD})$ & $10.6(2.2)$ & $10.7(2.1)$ & $10.3(2.3)$ \\
\hline Platelets count $(\mathrm{m} \pm \mathrm{SD})$ & $385.9(160.2)$ & $398.4(153.2)$ & $352.4(174.6)$ \\
\hline Neutrophil count $(\mathrm{m} \pm \mathrm{SD})$ & $5.0(2.6)$ & $5.0(2.7)$ & $5.1(2.5)$ \\
\hline Lymphocytes count $(m \pm S D)$ & $1.8(1.3)$ & $1.8(0.9)$ & $1.8(2.0)$ \\
\hline Creatinine count $(\mathrm{m} \pm \mathrm{SD})$ & $79.0(19.5)$ & $78.1(20.2)$ & $81.4(17.4)$ \\
\hline Live SGOT count $(\mathrm{m} \pm \mathrm{SD})$ & $29.4(5.2)$ & $29.0(4.0)$ & $30.4(7.4)$ \\
\hline Liver SGPT count $(\mathrm{m} \pm \mathrm{SD})$ & $32.0(4.9)$ & $32.1(4.4)$ & $31.9(6.2)$ \\
\hline Treatment adherence (Good) & $180(82.57)$ & $151(83.89)$ & $29(16.11)$ \\
\hline \multicolumn{4}{|l|}{ Treatment outcomes } \\
\hline Cured & $133(61.0)$ & & \\
\hline Lost-to follow-up & $16(7.3)$ & & \\
\hline Died & $32(14.7)$ & & \\
\hline Not Evaluated & $7(3.2)$ & & \\
\hline
\end{tabular}

$\mathrm{m}=$ mean; $\mathrm{SD}=$ standard deviation; $\mathrm{n}=$ number; $\%=$ percentage; for successful and unsuccessful, the column percentage was represented. 
Treatment outcomes

A total of $159(73 \%)$ patients had successful MDR-TB treatment at 9-months, $133(61 \%)$ were cured and $26(12 \%)$ completed treatment, while unsuccessful treatment outcomes was reported in $59(27 \%)$ patients. Among these patients with unsuccessful treatment outcomes, 32 (15\%) died, $16(7 \%)$ were lost to follow-up, 7 (3\%) were not evaluable, and 4 $(2 \%)$ had treatment failure (Table 1).

Predictors of successful treatment outcomes

In univariate logistic regression (Table 2), predictors associated with successful treatment outcomes were younger patients $(\mathrm{p}=0.0441 ; \mathrm{OR}=1.03)$, higher BMI more than 18.5 $\mathrm{kg} / \mathrm{m}^{2}(\mathrm{p}=0.0386 ; \mathrm{OR}=1.18)$, good adherence to treatment $(\mathrm{p}<0.0001 ;$ OR $=19.52)$, and the absence of clinical symptoms such as dyspnea $(p=0.0006 ;$ OR $=2.97)$, chest pain $(\mathrm{p}=0.0372 ; \mathrm{OR}=1.90)$, vomiting $(\mathrm{p}=0.0139 ; \mathrm{OR}=$
3.08), and depression ( $\mathrm{p}=0.0060 ; \mathrm{OR}=7.0)$. In addition, for patients with negative HIV $(\mathrm{p}=0.0552$; OR $=1.93)$, and patients who resided in an urban area $(\mathrm{p}=0.0591$; OR $=$ 2.22 ), we noted a trend association with the successful treatment outcomes. For the remaining predictors, we failed to show any association with the treatment success ( $p \geq$ 0.0600). The strongest contributing chance for higher successful treatment rates (Table 3) were higher BMI more than $18.5 \mathrm{~kg} / \mathrm{m}^{2}(\mathrm{p}=0.0253$; adjusted odds ratio $[\mathrm{aOR}]=$ $2.94)$, good adherence to treatment $(\mathrm{p}=<0.0001$; $\mathrm{aOR}=$ $33.92)$, normal platelets count $(\mathrm{p}=0.0053$; $\mathrm{aOR}=1.004)$, and the absence of clinical symptoms such as chest pain ( $\mathrm{p}=$ $0.0083 ; \mathrm{aOR}=3.19)$ and depression $(\mathrm{p}=0.0308 ; \mathrm{aOR}=$ 8.62). The corresponding chance of successful treatment was estimated using the following equation:

successful chance $=1 /\left(1+e^{-(-6.175+1.160 \times \text { Chest Pain }(\text { No })+2.153 \times \text { Depression }(\text { No })+1.078 \times \text { BMI }(>18.5)+3.523 \times \text { Adherence }(\text { Good })+0.004 \times \text { Platelets })}\right)$

Table 2. Predictors of successful MDR-TB treatment outcomes in Guinea, univariate analysis.

\begin{tabular}{|c|c|c|c|c|c|}
\hline Predictors & Estimate & SE & OR & $95 \%$ CI & p value \\
\hline Age (years) & -0.0263 & 0.0131 & 0.974 & $0.949-0.999$ & 0.0441 \\
\hline Gender (Male vs Femal) & 0.5523 & 0.3182 & 1.737 & $0.931-3.241$ & 0.0826 \\
\hline Residence (Urban vs rural) & 0.7953 & 0.4213 & 2.215 & $0.970-5.058$ & 0.0591 \\
\hline $\mathrm{BMI}(>18.5 \mathrm{vs} \leq 18.5)$ & 0.1663 & 0.0642 & 1.181 & $1.041-1.339$ & 0.0103 \\
\hline HIV (Yes vs No) & -0.6581 & 0.3432 & 0.518 & $0.264-1.015$ & 0.0552 \\
\hline History of TB treatment (Previously vs Newly) & 0.6936 & 0.3745 & 2.001 & $0.960-4.169$ & 0.0640 \\
\hline Sputum culture (Positive vs Negative) & 0.2877 & 0.4592 & 1.330 & $0.542-3.280$ & 0.5309 \\
\hline Lung Cavities (Yes vs No) & -0.2232 & 0.5620 & 0.800 & $0.266-2.407$ & 0.6913 \\
\hline Cough (Yes vs No) & 0.5586 & 0.5917 & 1.748 & $0.548-5.575$ & 0.3451 \\
\hline Dyspnea (Yes vs No) & -1.0900 & 0.3190 & 0.336 & $0.180-0.628$ & 0.0006 \\
\hline Chest pain (Yes vs No) & -0.6433 & 0.3088 & 0.526 & $0.287-0.963$ & 0.0372 \\
\hline Night sweat (Yes vs No) & 0.4189 & 0.3062 & 1.520 & $0.834-2.771$ & 0.1713 \\
\hline Nausea (Yes vs No) & -0.9327 & 0.5419 & 0.393 & $0.136-1.138$ & 0.0852 \\
\hline Vomiting (Yes vs No) & -1.1260 & 0.4576 & 0.324 & $0.132-0.795$ & 0.0139 \\
\hline Depression status (Yes vs No) & -1.9457 & 0.7084 & 0.143 & $0.036-0.573$ & 0.0060 \\
\hline Adherence (Yes vs No) & 2.9714 & 0.4466 & 19.519 & $8.135-46.835$ & $<.0001$ \\
\hline Leukocytes count & 0.0522 & 0.0516 & 1.054 & $0.952-1.166$ & 0.3114 \\
\hline Hemoglobin count & 0.0942 & 0.0732 & 1.099 & $0.952-1.268$ & 0.1982 \\
\hline Platelets count & 0.0018 & 0.0010 & 1.002 & $1.000-1.004$ & 0.0698 \\
\hline Neutrophil count & -0.00936 & 0.0597 & 0.991 & $0.881-1.114$ & 0.8755 \\
\hline Lymphocytes count & -0.0183 & 0.1222 & 0.982 & $0.773-1.248$ & 0.8807 \\
\hline Creatinine count & -0.0089 & 0.0080 & 0.991 & $0.976-1.007$ & 0.2650 \\
\hline Live SGOT count & -0.0538 & 0.0304 & 0.948 & $0.893-1.006$ & 0.0772 \\
\hline Liver SGPT count & 0.0053 & 0.0309 & 1.005 & $0.946-1.068$ & 0.8618 \\
\hline
\end{tabular}

$\mathrm{SE}=$ standard error, $\mathrm{OR}=$ odds ratio, $\mathrm{CI}=$ confidence interval, $\mathrm{BMI}=$ body mass index, $\mathrm{HIV}=$ human immunodeficiency virus.

The final model had good discriminatory capacity (Figure 2 ), the adjusted $c$-index for predicting successful treatment at 9-months was 0.848 (95\% bootstrap CI, $0.780-0.916)$, with the optimism from 1,000 samples of 0.042 corresponding to an optimism-corrected c-index of 0.806 .

Figure 2 Discriminative curve to predict the probability of successful MDR-TB treatment outcomes.

In addition, the calibration plots showed good agreement between the final model prediction and the actual predicted probability of successful treatment (Appendix, Figure A1).

The Hosmer and Lemeshow $X^{2}$ was 2.91 and the corresponding $p$-value was 0.94 . The uncertainties measured by the bootstrapping procedure in the internal validation were close to the estimated OR (Table 3), suggesting robustness of the final model. 
Table 3. Predictors of successful MDR-TB treatment outcomes in Guinea, multivariate analysis.

\begin{tabular}{llllll}
\hline \multirow{2}{*}{ Intercept } & Estimate (SE) & OR, 95\% CI & p value & $\begin{array}{l}\text { Maximum score in } \\
\text { nomogram }\end{array}$ & $\begin{array}{l}\text { Internal validation BOR, } \\
\mathbf{9 5 \%} \text { BCI }\end{array}$ \\
\cline { 2 - 6 } & $\mathbf{- 6 . 1 7 5}(\mathbf{1 . 3 4 5})$ & - & $<\mathbf{0 . 0 0 0 1}$ & - & - \\
\hline Depression (No vs Yes) & $2.153(0.997)$ & $8.619(1.220-60.895)$ & 0.0308 & 44 & $10.384(1.225-63.920)$ \\
BMI (>18.5 vs $\leq 18.5)$ & $1.079(0.482)$ & $2.942(1.143-7.569)$ & 0.0253 & 22 & $3.672(1.274-10.893)$ \\
Chest Pain (No vs Yes) & $1.160(0.439)$ & $3.192(1.348-7.561)$ & 0.0083 & 24 & $3.690(1.457-9.442)$ \\
Adherence (Yes vs No) & $3.523(0.561)$ & $33.915(11.287-101.905)$ & $<0.0001$ & 71 & $35.643(12.115-103.318)$ \\
Platelets count & $0.004(0.001)$ & $1.004(1.001-1.006)$ & 0.0053 & 100 & $1.004(1.001-1.007)$ \\
\hline
\end{tabular}

$\mathrm{SE}=$ standard error, $\mathrm{BMI}=$ body mass index, $\mathrm{OR}=$ odds ratio, $\mathrm{CI}=$ confidence interval, $\mathrm{BOR}=$ bootstrap odds ratio with 1,000 replications, $\mathrm{BCI}=$ bootstrap confidence interval.

The nomogram to predict the probability of 9-months successful treatment (Figure 1) showed that the normal plaquettes count and good adherence to treatment contributed the most strongly to the prognosis.

Whereas, the increased BMI more than $18.5 \mathrm{~kg} / \mathrm{m}^{2}$, and the absence of chest pain and depression at baseline had little effect on the probability of successful treatment. A row score was computed from the nomogram, and the patients were classified into four categories: low, moderate, intermediate, and high chance of successful treatment based on the thresholds defined in the Table 4.

Table 4. Discriminative performance of successful MDR-TB treatment outcomes in Guinea.

\begin{tabular}{|c|c|c|c|c|c|c|}
\hline Parameter & Value & Estimate & SE & OR & $95 \% \mathrm{CI}$ & p value \\
\hline Linear predictor score, mean (SD) & $1.32(1.80)$ & & & & & \\
\hline c-index by linear predictor score $(95 \% \mathrm{CI})$ & $0.848(0.780-0.916)$ & & & & & \\
\hline c-index by chance group $(95 \% \mathrm{CI})$ & $0.783(0.705-0.861)$ & & & & & \\
\hline Low chance $($ Total point $<135$ ) & $8 / 40(20 \%)$ & Reference & 0 & 1 & & \\
\hline Moderate chance $(\leq 135$ Total point $<160)$ & $35 / 42(83 \%)$ & 2.996 & 0.572 & 20.00 & $6.90-66.27$ & $<.0001$ \\
\hline Intermediate chance $(\leq 160$ Total point $<180)$ & $44 / 52(85 \%)$ & 3.091 & 0.551 & 22.50 & $7.89-69.57$ & $<.0001$ \\
\hline
\end{tabular}

$\mathrm{SE}=$ standard error, $\mathrm{OR}=$ odds ratio, $\mathrm{CI}=$ confidence interval, $\mathrm{SD}=$ standard deviation.

Using an optimal threshold of 135 points, the expected rate of successful treatment increased across chance categories, being $20,83,85$, and $88 \%$ respectively for low, moderate, intermediate, and high chance. The estimated performance measures based on the optimal threshold for predicting successful treatment were $95 \%$ for sensitivity, $58 \%$ for specificity, $86 \%$ for positive $\mathrm{PV}$, and $82 \%$ for negative PV. As an example of use of the nomogram and classification of patients as having high, intermediate, moderate, or low chance of successful treatment, a patient of any age or gender with good adherence to MDR-TB treatment (71 points), high BMI (22 points), no depression (44 points) or chest pain (24 points), and a plaquettes count of $300 / \mathrm{mm}^{3}$ ( 23 points) has 184 points, placing him in the intermediate chance group with a probability of 9-months successful treatment of $95 \%$ (Figure 1).

Points
Chest Pain
BMI
Adherence
Platelets count/mm3
Total Points
Linear Predictor

Figure 1. Prognostic nomograms to predict the probability of individual successful MDR-TB treatment outcomes. 


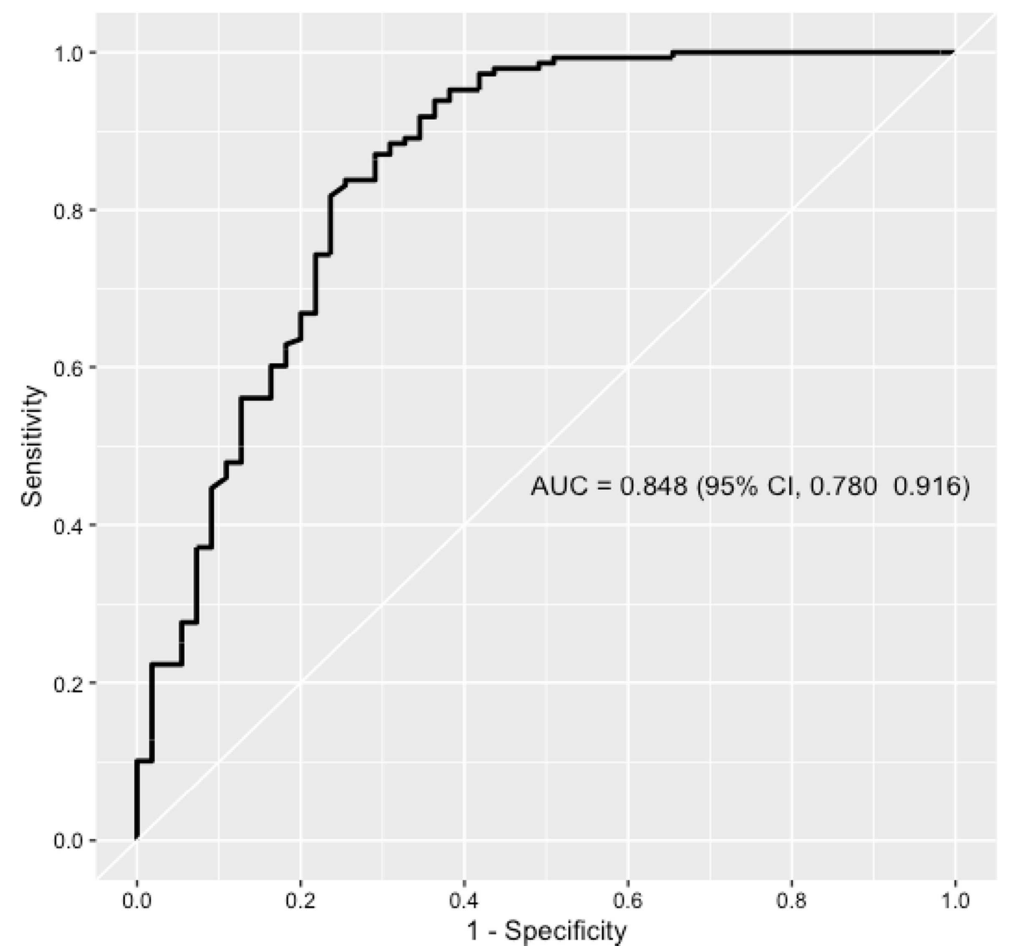

Figure 2. Discriminative curve to predict the probability of successful MDR-TB treatment outcomes.

AUC: area under curve and it $95 \%$ bootstrap confidence interval (CI) with 1,000 replications were the discriminative measure to predict the probability of successful MDR-TB treatment. The AUC value of 0.848 means that our model had a probability of $85 \%$ to detect patients who will be cured at the MDR-TB treatment end.

The $c$-index based on the overall performance of the nomogram raw score was $0.803(95 \% \mathrm{CI}, 0.724-0.881)$. From the linear predictor, the total points in nomogram and the corresponding probability of successful treatment can be obtained via the following formula: total point $=20.28318 \times$ linear predictor +122 , and successful chance $=$
$1 /\left(1+e^{-((\text {total points }-122) \times 0.04930194)}\right)$, where 122 is the scaling factors for generating only positive points scores and 0.04930194 is the linear predictor unit per point. To show how to estimate the chance of successful treatment across the point given by the nomogram see Table 5 .

Table 5. Point scoring for a successful treatment prediction score in MDR-TB patients.

\begin{tabular}{|c|c|c|c|c|c|}
\hline \multicolumn{2}{|c|}{ Plaquette count $/ \mathrm{mm}^{3}$} & \multicolumn{2}{|c|}{ Clinical symptoms } & \multirow{3}{*}{\multicolumn{2}{|c|}{$\begin{array}{l}\text { To convert the point score into chance of successful } \\
\text { treatment, look at the correspondence below }\end{array}$}} \\
\hline Range & points & Status & Points & & \\
\hline $\mathbf{0}$ & 0 & \multicolumn{2}{|l|}{ BMI $\left(\mathrm{kg} / \mathrm{m}^{2}\right)$} & & \\
\hline [0-100] & 8 & $\leq 18.5 \mathrm{~kg} / \mathrm{m}^{2}$ & 0 & Total points & Chance of successful treatment \\
\hline$[100-200]$ & 15 & $>18.5 \mathrm{~kg} / \mathrm{m}^{2}$ & 22 & $\leq 32$ & $\leq 1 \%$ \\
\hline$[200-300]$ & 23 & Depression st & & {$[32-46]$} & $2 \%$ \\
\hline$[300-400]$ & 31 & No & 44 & {$[46-66]$} & $5 \%$ \\
\hline [400-500] & 38 & Yes & 0 & {$[66-81]$} & $10 \%$ \\
\hline$[600-700]$ & 54 & No & 24 & [97-108] & $30 \%$ \\
\hline$[700-800]$ & 62 & Yes & 0 & [108 - 117] & $40 \%$ \\
\hline [800-900] & 69 & & & {$[117-125]$} & $50 \%$ \\
\hline [900-1000] & 77 & & & {$[125-133]$} & $60 \%$ \\
\hline$[1000-1100]$ & 85 & & & [133 - 142] & $70 \%$ \\
\hline$[1100-1200]$ & 92 & & & [142 - 153] & $80 \%$ \\
\hline \multirow[t]{2}{*}{$\geq 1200$} & 100 & & & {$[153-170]$} & $90 \%$ \\
\hline & & & & [204 - 218] & $99 \%$ \\
\hline Adherence status & & \multirow{4}{*}{\multicolumn{2}{|c|}{$\begin{array}{l}\text { Sum up the points across the all } 5 \text { factors above to obtain } \\
\text { the total point score }\end{array}$}} & $>218$ & $100 \%$ \\
\hline Status & Points & & & & \\
\hline Poor & 0 & & & & \\
\hline Good & 71 & & & & \\
\hline
\end{tabular}

BMI: body mass index by divided weight by the square of height $(\mathrm{m})$. 


\section{Discussion}

In this study, we identified predictors and derived prognosis nomogram of 9-months MDR-TB treatment success.

During the treatment period, the success rate was $73 \%$, which was slightly below than the success rate recommended by the WHO (75\%). The high rate of mortality $(15 \%)$ and lost to follow-up (7\%) due to the several factors such as delay diagnosis or the HIV infection could be explained the low success rate in our sample. Compared to the recently published studies [4-10], our success rate was higher than those reported in Morocco (53.5\%) [5], Brazil (60\%) [4], and in Bashkortostan region of Russia (67\%) [11], comparable to those reported in Baluchistan province of Pakistan (71.6\%) [8] and Tanzania (75.7\%) [6], and lower than those reported, in Yemen (77.4\%) [7], Eastern Taiwan (78.4\%) [9], and Rwanda (87.3\%) [10]. Possible reasons for these discrepancies are selection bias, differences in MDR-TB regimens, genetic background, and differences between health-care systems.

After 9-months MDR-TB treatment, 7\% of our patients were lost to follow-up, which was higher to those reported in Rwanda (0.6\%) [10], comparable to those reported in Baluchistan province of Pakistan (7.5\%) [8], and lower than those reported recently in several studies [4, 6, 9] as in Morocco where $34.6 \%$ of patients lost to follow-up [5]. Poor adherence to treatment due to the adverse drug reactions was an important factor associated with loss-to-follow-up in our report. Moreover, the higher treatment costs, long duration of treatment, poor knowledge/understanding of MDR-TB, high level of poverty, a low economic status, low family support, and failure health services were previously reported as an important predictors associated with loss-to-follow-up [18, 19]. These high rates of loss of follow-up are alarming for health systems, causing a danger to the population, possibly increasing the incidence of extensively drug-resistant tuberculosis. The failure rate was $2 \%$ in our study, which was higher to those reported in Rwanda $(0.6 \%)$ [10], Tanzania (0.6\%) [6], and Baluchistan province of Pakistan (1.1\%) [8], but lower than those found in Yemen (3.5\%) [7], Eastern Taiwan (5.4\%) [9], Morocco (6.9\%) [5], and Brazil (9\%) [4].

We identified the higher BMI, good adherence to treatment, absence of depression and chest pain, and normal platelets count as independent predictors associated with successful MDR-TB treatment. Similar results [7, 8] have been found that the lower BMI $\left(\leq 18.5 \mathrm{~kg} / \mathrm{m}^{2}\right)$ is an independent factor for unsuccessful treatment in MDR-TB patients. It has been suggested that the lower immunity, poor absorption from gastrointestinal tract or inadequate dosing drugs in underweight patients are possible explanation of association between MDR-TB treatment outcomes and BMI [20].

Not surprisingly, good adherence to treatment was associated with higher success rate with an adjusted odds ratio $(\mathrm{aOR})$ of 34 . In our sample only $17 \%$ of patients were poor adherents during treatment, among them $80 \%$ are classified as unsuccessful treatment outcomes. The poor adherence rate reported in this study was lower than those found in recent meta-analysis $(20 \%)$ conducted in migrant population [21] where the social risk factors were the main reason. In addition, although drug adverse reactions may explain non-adherence to treatment, we are convinced that the multiplication of awareness campaigns during treatment can help reduce these rates.

The absence of anxious and/or depression was found as important factor to MDR-TB treatment outcomes, nodepressed patients had an increase chance of being cured or complete treatment $(\mathrm{aOR}=8.9)$. Although we have captured depression by asking patients whether they are depressed or not, it would be important to confirm the association between depression and treatment success by measuring it through validated scales such as the PHQ-9 scale [22]. This association suggests the conducting of prospective studies to evaluate the efficacy of psychotherapy management during the treatment of MDR-TB patients.

Chest pain and normal plaquette count were found to be associated with treatment outcomes, patients with no chest pain and patients who had normal platelets count at baseline had an increase chance of being successful treatment $(\mathrm{aOR}=$ 3.2 and 1.004 respectively). Although more frequently rifampicin and rarely pyrazinamide induce thrombocytopenia $\left(<150,000 \mathrm{~mm}^{3}\right)$, at inclusion only $8 \%$ of our patients had platelets $<150,000 \mathrm{~mm}^{3}$.

In univariate analysis, we found that younger patients and negative HIV patients had an increase chance of being successful treatment ( $\mathrm{OR}=1.03$ and 1.93 respectively). Similar results have been reported by studies conducted elsewhere [8, 10, 12]. Nevertheless, others factors including non-use alcohol misuse [12], non-smoker [5], acid fast bacilli negative sputum [4, 7,9], first episode of MDR-TB $[4,9]$, cavitary lesions on X-ray $[6,7]$, resistance to streptomycin and Ethambutol [6] had been reported as independent predictors to successful treatment. While, we not found any association between cavitary lesions and treatment outcomes. These discordant finding suggest the conduct of an international meta-analysis on individual data to definitively identify factors associated with successful treatment.

Based on the number of easily accessible predictors identified in the final logistic model, we constructed a nomogram in a rigorous methodology framework that allowed prediction of individual treatment success with high precision. The nomogram had good discriminatory capacity, and there was good agreement between the nomogram prediction and actual rates of success. Moreover, using the ROC curve methodology, the threshold of 135 points was identified to be the optimal cut-off to select patients with high chance of being successful treatment. However, the nomogram needs to be externally validated on independent samples including non-Guinean patients to establish the generalisability of the model. While, some missing parameters such as diabetes status, smoking and alcohol use, and biomarkers were limits of this study, they might be 
further used to improve nomogram.

\section{Conclusion}

The current study allowed to develop and internally validate a prognosis nomogram to estimate the chance of successful treatment in MDR-TB patients. This nomogram was developed from the easily accessible predictors including body weight, adherence to treatment, platelets count, chest pain, and depression status. Considering these predictors could be improved the clinical outcomes and the management strategy of MDR-TB by the strengthening of patient monitoring during treatment, considering the psychological state of patients through psychological consultation, and initiating new food programs. Our nomogram provides more information to help with patient selection and stratification for future clinical trials.

\section{Abbreviations}

BMI: body mass index; BOR: bootstrap odds ratio, BCI: bootstrap confidence interval; $\mathrm{CI}$ : confidence interval; $\mathrm{CRF}$ : case report form; DST: drug susceptibility testing; HIV: human immunodeficiency virus; H: Hosmer; INH: isoniazid; L: Lemeshow; MDR-TB: Multidrug-resistant tuberculosis; PDC: proportion of days covered; PV: predictive value; OR: odds ratio; ROC: receiver-operating characteristic; SD: standard deviation; SE: standard error; TB: tuberculosis; WHO: World Health Organization.

\section{Declarations}

\section{Ethics Approval and Consent to Participate}

The study was approved by the National Ethics Committee for Heath Research (NECHR) reattached to the Ministry of Health (Conakry, Guinea). The study was based in accordance to the Declaration of Helsinki, and the confidentiality of the data was guaranteed.

\section{Consent for Publication}

Not applicable.

\section{Availability of Data and Materials}

The data is available upon authors request.

\section{Competing Interests}

The authors declare that they have no competing interest in relation with this work.

\section{Funding}

None

\section{Author's Contributions}

AD conceived the study design, analysed the data, and drafted the manuscript, BDD contributed to the conception, organization the research project, supervision data collection, drafting and critical revision to manuscript, MD collected data and commented the manuscript, SC, LMC, OYS, contributed to the conception, organization the research project, and critical revision to manuscript, BB, MAS, AOB, THD, LM, and MAD commented the manuscript. All authors approved the final version of the manuscript.

\section{Appendix}

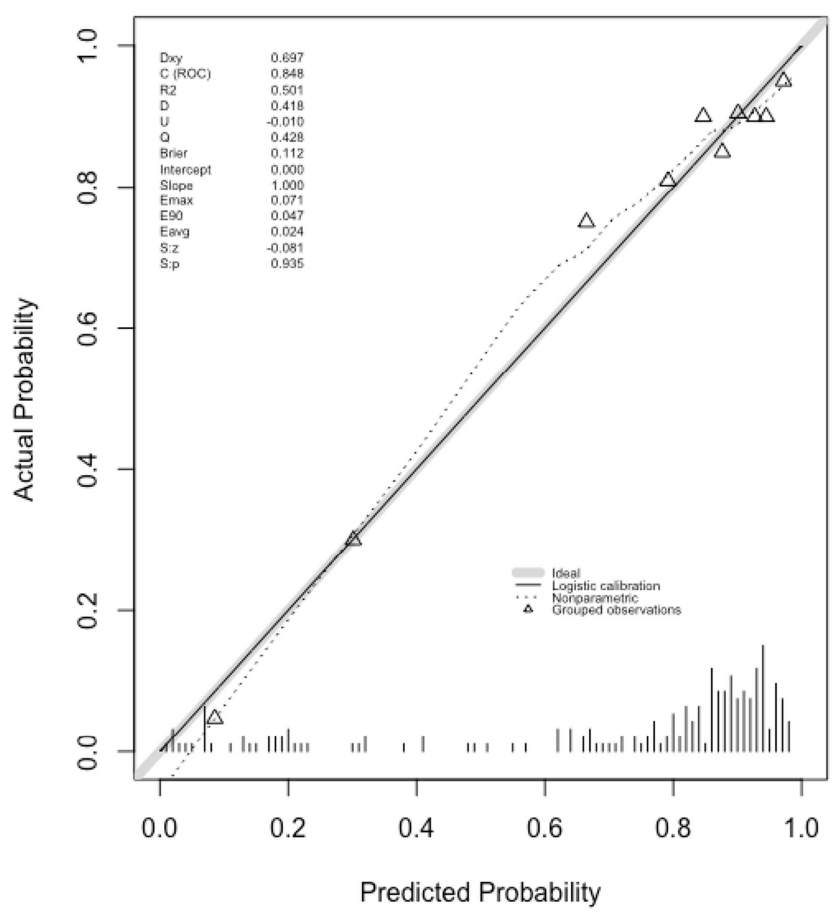

Figure A1. Prognostic model validation of successful MDR-TB treatment outcomes.

Clinical nomogram for patients starting treatment for MDR-TB, estimating the probability of successful treatment. Instruction for using the nomogram: Locate the patient's chest pain on the Chest pain axis. Draw a straight vertical line up to the Points axis to determine how many points the chest pain of the patient contribute towards the predicted probability of successful treatment. Repeat this process for depression status, BMI, treatment adherence, and platelets normal count. Sum the points from each of the predictor to get the total points. Locate the patient's total points on the Total points axis. Draw a straight line down to the Probability of successful MDR-TB treatment axis to determine the probability of successful during treatment for that patient. The chance of successful treatment is provided as a proportion and should be multiplied by 100 to obtain the value in percentage.

\section{Acknowledgements}

We thank the National Tuberculosis Control Program for their collaboration, and Action Damien Foundation for their patients support during treatment. 


\section{References}

[1] Caminero JA. Multidrug-resistant tuberculosis: epidemiology, risk factors and case finding. Int J Tuberc Lung Dis Off J Int Union Tuberc Lung Dis. 2010; 14 (4): 382-90.

[2] World Health Organization: Global tuberculosis report 2017. Geneva, Swizerland: WHO press, 2017.

[3] National Tuberculosis Control Program Guinea: Annual report of TB control activity, 2018.

[4] Bastos ML, Cosme LB, Fregona G, do Prado TN, Bertolde AI, Zandonade E, et al. Treatment outcomes of MDR-tuberculosis patients in Brazil: a retrospective cohort analysis. BMC Infect Dis. $2017 ; 17$ (1).

[5] El Hamdouni M, Bourkadi JE, Benamor J, Hassar M, Cherrah Y, Ahid S. Treatment outcomes of drug resistant tuberculosis patients in Morocco: multi-centric prospective study. BMC Infect Dis. 2019; 19 (1): 316.

[6] Leveri TH, Lekule I, Mollel E, Lyamuya F, Kilonzo K. Predictors of Treatment Outcomes among Multidrug Resistant Tuberculosis Patients in Tanzania. Tuberc Res Treat.; 2019: 110.

[7] Jaber AAS, Ibrahim B. Evaluation of risk factors associated with drug-resistant tuberculosis in Yemen: data from centres with high drug resistance. BMC Infect Dis. 2019; 19 (1): 464.

[8] Khan I, Ahmad N, Khan S, Muhammad S, Ahmad Khan S, Ahmad I, et al. Evaluation of treatment outcomes and factors associated with unsuccessful outcomes in multidrug resistant tuberculosis patients in Baluchistan province of Pakistan. J Infect Public Health. 2019;

[9] Lin C-B, Sun H-C, Chiang C-Y, Wu C-W, Chou H-W, Tang T-Q, et al. Treatment outcomes for multidrug-resistant tuberculosis in Eastern Taiwan. Tzu Chi Med J. 2019; 31 (1): 35.

[10] Muvunyi CM, Ngabonziza JCS, Uwimana I, Harelimana JDD, Mucyo Y, Sebatunzi OR, et al. Highly successful treatment outcome of multidrug-resistant and genetic diversity of multidrug-resistant Mycobacterium tuberculosis strains in Rwanda. Trop Med Int Health. 2019; 24 (7): 879-887.

[11] Yunusbaeva M, Borodina L, Alekseev P, Davydov R, Yunusbaev U, Sharipov R, et al. Treatment efficacy of drug- resistant tuberculosis in Bashkortostan, Russia: A retrospective cohort study. Int J Infect Dis. 2019; 81: 203-9.

[12] Samuels JP, Sood A, Campbell JR, Ahmad Khan F, Johnston JC. Comorbidities and treatment outcomes in multidrug resistant tuberculosis: a systematic review and meta-analysis. Sci Rep. 2018; 8 (1).

[13] Falzon D, Schünemann HJ, Harausz E, González-Angulo L, Lienhardt C, Jaramillo E, et al. World Health Organization treatment guidelines for drug-resistant tuberculosis, 2016 update. Eur Respir J. 2017; 49 (3): 1602308.

[14] Hansen RA, Kim MM, Song L, Tu W, Wu J, Murray MD. Adherence: Comparison of Methods to Assess Medication Adherence and Classify Nonadherence. Ann Pharmacother. 2009; 43 (3): 413-22.

[15] WHO: Definitions and reporting framework for tuberculosis 2013 revision (updated 2014). Geneva: Switzerland World Health Organization; 2013.

[16] Hosmer DW, Lemeshow S. Applied logistic regression [Internet]. New York; Toronto: John Wiley \& Sons; 2005.

[17] Youden WJ. Index for rating diagnostic tests. Cancer. 1950; 3 (1): $32-5$.

[18] Kliiman K, Altraja A. Predictors of poor treatment outcome in multi- and extensively drug-resistant pulmonary TB. Eur Respir J. 2009; 33 (5): 1085-94.

[19] Yew WW, Chan CK, Chau CH, Tam CM, Leung CC, Wong PC, et al. Outcomes of Patients With Multidrug-Resistant Pulmonary Tuberculosis Treated With Ofloxacin/Levofloxacin-Containing Regimens. Chest. 2000; 117 (3): 744-51.

[20] Byrd, Jr. RP, Mehta JB, Roy TM. Malnutrition and Pulmonary Tuberculosis. Clin Infect Dis. 2002; 35 (5): 634-5.

[21] Nellums LB, Rustage K, Hargreaves S, Friedland JS. Multidrug-resistant tuberculosis treatment adherence in migrants: a systematic review and meta-analysis. BMC Med. 2018; 16 (1).

[22] Spitzer RL, Kroenke K, Williams JB. Validation and utility of a self-report version of PRIME-MD: the PHQ primary care study. Primary Care Evaluation of Mental Disorders. Patient Health Questionnaire. JAMA. 1999; 282 (18): 1737-44. 